\title{
Juventud, academia y empleo. Análisis de una desconexión
}

Youth, academy and employment. Analysis of a disconnection

Carlos De Domingo Soler ${ }^{1}$, Mónica Naveda Jácome ${ }^{2}$, Marco Antonio Rodríguez Proaño ${ }^{3}$, María Gabriela Muñoz Pumagualle ${ }^{4}$

INFORMACIÓN DEL

\section{ARTÍCULO}

Fecha de recepción: 21 de Marzo de 2020.

Fecha de aceptación: 22 de Junio de 2020 .

1 Máster en Acceso a la Abogacía, Universidad de Nebrija. Docenteinvestigador, Universidad de Los Hemisferios-Ecuador.

E-mail:

carlosdedomingosoler@gmail.com. Código ORCID:

https://orcid.org/0000-0003-4656-9625

${ }^{2}$ Magíster en Corrupción y Estado de Derecho, Universidad de Salamanca. Ex inspectora de trabajo, Ministerio del Trabajo-Ecuador.

E-mail: mona_naveda@hotmail.com

Código ORCID

https://orcid.org/0000-0001-8909-4186

3 Maestría en Derecho mención Derecho del Mercado, Universidad Andina Simón Bolívar. Docenteinvestigador, Universidad Andina Simón Bolívar-Ecuador.

E-mail: marco.rodriguez6@icloud.com Código ORCID:

https://orcid.org/0000-0001-5241-9223

\begin{tabular}{l}
\hline $4 \quad$ Máster en Comunicación, \\
Universidad de las Américas. \\
Docente-investigadora, IDE Business \\
School-Ecuador. \\
E-mail: gm41271@gmail.com. \\
Código ORCID: \\
https://orcid.org/0000-0002-5842-2738
\end{tabular}

CITACIÓN: De Domingo Soler, C., Naveda Jácome,M., Rodríguez Proaño, M.A.,\& Muñoz Pumagualle, M.G.(2020). Juventud, academia y empleo. Análisis de una desconexión. Podium, 37, 129-146. doi:10.31095/podium.2020.37.9

ENLACE DOI:

http://dx.doi.org/10.31095/podium.202 0.37 .9

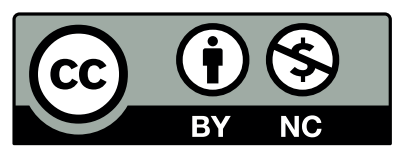

\section{Resumen}

En el imaginario colectivo ecuatoriano predomina la idea de que, para un desenvolvimiento laboral competitivo es indispensable cursar estudios universitarios. El objetivo del presente trabajo es realizar una aproximación teórica a la actual relación entre la academia y la empresa en el Ecuador, estableciendo como marco temporal el año 2019. Para ello se adopta un enfoque interdisciplinar, estadístico y sociológico, y se emplea una metodología cuantitativa y cualitativa-interpretativa de las estadísticas oficiales, aportándose entrevistas en profundidad y de estructura temática. Como conclusión se sostiene la existencia de una desconexión entre la academia y los sectores productivos -especialmente perjudicial para el segmento juvenil no inserto en la esfera laboral-, la cual hace peligrar la consecución por parte del Ecuador del ODS $8^{\circ}$, toda vez que, según las últimas mediciones oficiales, la tasa de desempleo juvenil alcanza el 9\% y apenas el $35 \%$ de jóvenes con titulación superior posee empleos adecuados.

\section{Palabras Clave:}

Demanda laboral, empleo, jóvenes, Objetivos de Desarrollo Sostenible, oferta laboral, politica pública relacionada.

Clasificación JEL: J1, J2.

\begin{abstract}
In the Ecuadorian collective-imaginary, the idea that in order to achieve a competitive work development it is essential to pursue University studies prevails. The objective of this work is to carry out a theoretical approach to the current Academy-Labor relationship in Ecuador, taking the year 2019 as the frame. To do this, it starts from an interdisciplinary, statistical and sociological approach, while a quantitative and qualitative-interpretive methodology of official statistics and in-depth interview -with thematic structure- are used. As conclusion, the existence of a disconnection between the Academy and the productive sectors is sustainedwhich especially harms que segment of young people who have not yet managed to get involved in the labor field-, endangering the achievement of the United Nations 8th SDG by Ecuador, because of, according to the latest official measurements, the youth unemployment rate reaches $9 \%$ and only $35 \%$ of young people with higher degrees have adequate jobs.
\end{abstract}

Keywords:

Labor Demand, Employment, Youth, Sustainable Development Goals, Labor Supply, Related Public Policy.

JEL Classification: J1, J2.

\footnotetext{
PODIUM No. 37, Junio 2020, pp. 129-146

(C) Universidad Espíritu Santo - UEES

ISSN: 1390-5473 e-ISSN: 2588-0969
} 


\section{Introducción}

El último reporte de la Encuesta Nacional de Empleo, Desempleo y Subempleo (ENEMDU), con información actualizada a diciembre de $2019^{5}$, revela que mientras 12.402 .565 personas se encuentran en edad de trabajar $^{6}$ solo 3.146.297 cuentan con un empleo adecuado $^{7}$ (INEC, 2019a). Para obtener datos específicos sobre la relación entre las cifras de empleo y los distintos niveles de instrucción académica se debe acudir a la ENEMDU Acumulada de 2018 y a la tabulación final de la ENEMDU de 2019 . En 2018 la sumatoria de las tasas de desempleo, subempleo ${ }^{9}$ y empleo no remunerado en personas con nivel de instrucción superior alcanzó el $27,9 \%$ en hombres y el $33,5 \%$ en mujeres (INEC, 2018), mientras que en 2019 el INEC únicamente utilizó la variable clasificatoria de educación superior para medir la tasa de empleo adecuado, la cual fue del $35 \%$

\footnotetext{
${ }^{5}$ La publicación de la ENEMDU actualizada a marzo de 2020 estaba prevista para finales de abril del mismo año. No obstante, la crisis sanitaria obligó al INEC a postergar indefinidamente su publicación.

${ }^{6}$ Según la relación de definiciones de la ENEMDU, la categoría población en edad de trabajar "comprende a todas las personas de 15 y más" (INEC, 2019, p.5).

${ }^{7}$ Como es sabido, la noción de empleo adecuado -“condición laboral en la cual las personas satisfacen ciertas condiciones mínimas" (INEC, 2019b, p. 7), y en la que se "perciben ingresos laborales iguales o superiores al salario mínimo, trabajan igual o más de 40 horas a la semana" (INEC, 2019ª, p. 5)-dista mucho del cumplimiento de las exigencias del trabajo decente o empleo digno (OIT, 1999).

8 Por cuanto los ciclos económicos son sistémicos y estructurales, no puede afirmarse que la cuestión que aquí se expone carezca de causales ajenas al ámbito educativo (datos macroeconómicos regionales, balanza, normativa laboral, costo del despido intempestivo), o inherentes a él, como la propia calidad de la educación superior o la conocida "fuga de cerebros" al extranjero.

${ }^{9}$ Para una idea general de su implicación socioeconómica, las personas subempleadas reciben un promedio mensual de $149,62 \$$, dándose el 19,9\% de estos empleos en el sector formal
} (INEC, 2019b).
(INEC, 2019b $)^{10}$. Durante las dos últimas décadas mucho se ha escrito sobre la interdependencia entre formación superior y acceso al mercado laboral (Guerrero, 1999; Jiménez, 2009; Planas Coll y Enciso Ávila, 2014; Brzinsky-Fay, 2017), hasta el punto, incluso, de configurarse como una suerte de mantra en el imaginario colectivo. Si bien hay quienes defienden el determinismo de la absorción laboral como sucesora natural de la educación superior, también existen corrientes críticas, tanto en Europa como en América Latina, que ponen en tela de juicio la presunta organicidad entre estudios y empleo (Fernández Enguita, 1992; Mungaray, 2001; De Ibarrola, 2005; Márquez, 2011).

Mediante esta investigación se pretende indagar en la relación existente entre la educación superior ecuatoriana y las tasas de las distintas tipologías de empleo que se desprenden de la ENEMDU de 2019. Más concretamente, se aspira a responder si la posesión de un título de educación superior es un elemento diferencial a la hora de poseer un empleo adecuado, o, por el contrario, la problemática del empleo no adecuado es transversal a los distintos niveles de instrucción; $y$, de probarse dicha transversalidad, ¿cuál es el papel de las Instituciones de Educación Superior

\footnotetext{
${ }^{10}$ Nótese que entre 2018 y 2019 el INEC aplicó de forma desigual dicha variable clasificatoria. Resulta curioso, por otro lado, que se observen inconsistencias entre las cifras de ambos años. En efecto, la ENEMDU Acumulada de 2018 ubicó el porcentaje de empleo adecuado con educación superior en $70,1 \%$ en hombres y $65,3 \%$ en mujeres (INEC, 2018). Por su parte, el histórico del tabulado de la ENEMDU de 2019 indica que en diciembre de 2018 la tasa de empleo adecuado con educación superior, sin distinción de género, era del 35\% (INEC, 2019b).
} 
(IES), los segmentos productivos y el Estado?, ¿y cuál la afectación en las vidas de la población juvenil con estudios terciarios?

Se justifica esta investigación a raíz de la necesidad que afronta el Ecuador -más ahora, habida cuenta del golpe que, para las predicciones de escaso crecimiento económico (ASOBANCA, 2020a; BCE, 2020), puede causar la actual crisis sanitaria, con expectativas de variación del PIB de entre $-4,3 \%$ y $-5,3 \%$ (ASOBANCA, 2020b)- de repensar el relacionamiento entre la Academia y el sector productivo. Para abordar esta cuestión se recurrirá a una perspectiva interdisciplinaria, sociológica y de política pública, empleando como metodología el análisis de las estadísticas oficiales de distintos organismos públicos ecuatorianos. Sin embargo, surge como principal limitación la falta de especificidad de los datos estadísticos de empleo en función del nivel de instrucción y la ausencia de estudios consolidados de empleabilidad por parte de la Secretaría de Educación Superior, Ciencia, Tecnología e Innovación (SENESCYT).

El presente texto se estructura de la siguiente forma. En el segundo apartado se hace una breve revisión a la literatura sobre juventud, empleo y formación superior. A su vez, se identifica el marco normativo y los posibles objetivos estratégicos afectados, tanto respecto al Plan Nacional de Desarrollo 2017-2021 (SENPLADES, 2017) como a los ODS (Naciones Unidas, 2018). En el tercer apartado, se describe la metodología y los enfoques en los que se basa la investigación. En el apartado cuarto se exponen los hechos y los datos estadísticos que facultan a concluir, en el último apartado, que existe una desconexión entre las competencias curriculares ofertadas por las IES y las competencias profesionales demandadas por el sector productivo ecuatoriano.

\section{Revisión de literatura}

Este trabajo versa sobre juventud, absorción laboral joven y la relación entre empleo y educación superior. Por ende, y para mayor aprovechamiento, la revisión de literatura expone individualmente cada una de las temáticas mencionadas, con el objeto de, en primera instancia, plantear cada materia de forma endogámica para, en última instancia, conjuntarlas en el apartado de resultados como un único planteamiento general y rizomático.

El artículo primero de la Ley de la Juventud (LJ) es preciso al considerar joven a todas las personas comprendidas entre 18 y 29 años. A pesar de ello, los rangos de edad de las distintas categorías medidas por el INEC (2018, 2019a y 2019b) no reflejan en una única variable consolidada la delimitación etaria de la LJ. ${ }^{11}$ Ello distorsiona toda posible información sobre las tasas de empleo propiamente juveniles, a más que

\footnotetext{
${ }^{11}$ En la ENEMDU, el INEC contribuye al desconocimiento de las tasas laborales específicas de la población joven, habida cuenta que utiliza dos rangos de edades jóvenes (de 15 a 24 años, y de 25 a 34 años). Es decir, a la luz de la LJ el rango 25-34 contiene 4 años del espectro joven (de 25 a 29) pero excede en 5 años el límite del espectro (de 29 a 34). Lo mismo ocurre con la estadística de dinámica laboral y empresarial (INEC, 2017b).
} 
dificulta un eventual contraste con el resto de los instrumentos públicos de medición ${ }^{12}$. Si se repasa el conjunto de investigaciones científicas realizadas en los últimos años sobre empleo juvenil (Olmedo, 2018; Mullo y Marcatoma, 2018; Petter y Moreno, 2019; Quispe, Sánchez y Arellano, 2019) se comprueba que, aún habiendo contribuido valiosamente con una radiografía multidimensional de la problemática, estas no analizaron críticamente la divergencia entre los rangos de edad, la categoría jurídica y sociológica "joven" y la edad de los sujetos pasivos de las políticas públicas especializadas, como se pretende aquí.

Sostienen varios estudios que los miembros de las generaciones millennial y Z están instalados en el pesimismo (Londoño, 2009; González Arratia y Valdez Medina, 2012; M\&C Saatchi, 2018; Deloitte, 2019). Si bien este pesimismo es antropológico, constitutivo de la cultura occidental moderna (BID, 2017) y derivado de "supuestos cognitivos de la personalidad" (González Arratia y Valdez Medina, 2012, p. 208), posee fuertes implicaciones empresariales y laborales, toda vez que interviene negativamente en el desarrollo psicológico de los jóvenes y sus estados de ánimo (Seligman, 1999). Sobre el pesimismo de la juventud ecuatoriana apenas se ha escrito entre 2006 y 2019 , y aún así se hizo de forma escueta y

\footnotetext{
12 Por ejemplo, la estadística de matriculación en educación superior únicamente tiene en cuenta el rango de 18 a 24 años (SENESCYT, 2018b). Sin embargo, sí utiliza el rango de 18 a 29 años con respecto a la medición del acceso a la educación superior por razones económicas (SENESCYT, 2018b).
}

aplicada a la calidad futura del trabajo y al emprendimiento (Espinosa y Esteves, 2006; Zambrano y Lasio, 2019). El pesimismo no es gratuito ni voluntario, sino resultado de crisis económicas, destrucción masiva de puestos de trabajo y auge de la precariedad laboral (Cano, 2014). Tampoco sería descabellado imaginar que la esperanza juvenil de aspirar a un empleo adecuado se ha visto mermada a causa de la actual emergencia sanitaria, acrecentando la ya de por sí palmaria crisis del empleo de los jóvenes (OIT, 2012).

La incidencia demográfica de la juventud en el Ecuador es notable. Los jóvenes representan el $20 \%$ de la población total (Secretaría Técnica de Juventudes, 2018). Sin embargo, del total de personas empleadas el $14,7 \%$ y el $22 \%$ tienen entre 15 y 24 años y 25 y 34 respectivamente (INEC, 2019b), mientras que en 2019 el rango estadístico de 15 a 34 años apenas alcanzó el $35,1 \%$ del empleo adecuado total (INEC, 2019b) ${ }^{13}$, un $3,8 \%$ menos que en 2017 (Secretaría Técnica de Juventudes, 2018). En cuanto a la tasa de desempleo, en 2019 el desempleo juvenil representó el $67,2 \%$ del desempleo total, con un $71,4 \%$ de cesantes y solo un $28,6 \%$ de personas sin experiencia laboral previa (INEC, $2019 b)^{14}$. Ahora bien, ¿cómo se vinculan estas estadísticas con el nivel de

\footnotetext{
13 A excepción del rango de 65 años en adelante, con una incidencia del 3,6\%, el grueso de empleo adecuado se encuentra en los rangos de 35 a 64 años, con un 61,3\% (INEC, 2019b: $\S 4.2)$.

${ }^{14}$ El BID realizó un estudio en Bolivia en 2015, en el que concluyó que los principales motivos de no contratación de nuevo personal calificado eran la carencia de habilidades, en un $31 \%$, y la carencia de experiencia, en un $25 \%$.
} 
instrucción? En las primeras páginas se afirmó, en base a la data del INEC de 2019, que sólo el $35 \%$ de personas poseedoras de un título de educación superior cuentan con empleo adecuado, es decir, el $64 \%$ de empleos adecuados se encuentran en posesión de personas con educación básica, media o bachiller (INEC, 2019b). Ciertamente, la educación superior produce "egresados con mejores oportunidades laborales y mayores defensas frente al desempleo" (Mungaray, 2001, p. 62). La propia OECD defiende que "los individuos con un nivel educativo alto generalmente [...] tienen mayores tasas de empleo y tienen mayores ingresos relativos" (2015, p. 9). Ahora bien, en el contexto de la expansión educativa (OECD, 2017), la tasa de entrada a la educación superior ecuatoriana ha aumentado paulatinamente desde el año 2000 (Ferreyra, Avitabile, Botero Álvarez, Haimovich, y Urzúa, 2017). Como muestra la SENESCYT, la tasa bruta de matrícula en IES presenta una tendencia creciente desde 2012, y se esperaba, antes de la crisis sanitaria, que en 2021 alcanzase el 31,21\% (2018b). En contraposición, como muestran las cifras del INEC, el empleo adecuado se ha ido deteriorando desde 2014 (2017a). Esto es, a medida que se han alcanzado nuevas cotas de estudiantes de educación superior, aumentándose en cada ejercicio la inversión (SENESCYT, 2018a), la tasa de empleo adecuado ha disminuido. Desde luego, la diatriba entre estudiar o no programas de educación superior como medida de "protección frente al desempleo" plantea lecturas discordantes. En materia de empleo asalariado, en 2015 y 2016 la tasa neta de entrada al sistema de seguridad social dejó un saldo de $-4,13 \%$, afectando principalmente a los más jóvenes (de 15 a 34 años) y a aquellos sin educación superior (INEC, 2017a), sin embargo, en 2017 el 70,6\% de empleados carecían de títulos de educación superior (INEC, 2017b).

En contra del mantra de la educación superior como diferencial para acceder al empleo adecuado, Bonilla (2018) reconoció que, pese a que los estudiantes tienen la convicción de que al estudiar y terminar esas carreras tienen altas expectativas de estabilidad laboral, [ello] no es necesariamente cierto. Las causas del desempleo juvenil son tan sistémicas como particulares, estrechamente dependientes de los ciclos económicos y otros factores [macroeconómicos] que no se pueden controlar a nivel nacional. Lógicamente, el papel de la planificación pública es indispensable. Es necesario diseñar políticas públicas integrales que consideren la transición entre instituciones académicas y trabajo. Como es sabido, en economías no centralizadas la iniciativa privada posee amplias capacidades para dinamizar la sociedad. Concretamente, mediante el empleo de jóvenes estudiantes, la empresa privada tiene ante sí grandes oportunidades (Quispe, Sánchez y Arellano, 2019). En el Ecuador la importancia económica de la iniciativa privada es innegable, con un total de 899.208 empresas contabilizadas en 2018 (INEC, 2019c). No en vano, en 2015 el $72 \%$ de personas asalariadas pertenecían al sector privado (INEC, 2017b) y en 2019 el 81,3\% del empleo adecuado total se encontraba en la esfera privada (INEC, 2019b). Para generar mayor sinergia 
entre juventud y empleo, academia y sector productivo, es necesario permitir a las IES y a la empresa, según Ayala Mora (2018) producir los recursos que el Ecuador necesita para que la sociedad avance y se desarrolle en términos comparativos, sugerencia que se antoja imposible sin la condición irrefutable de la autonomía universitaria y la disponibilidad de los sectores empresariales para aportar a la universidad. Ello es indispensable para aspirar a un school-to-work transition system, que considere a las instituciones educativas y al mercado laboral conjuntamente, esto es, siguiendo a Brzkinsky-Fay (2017) y su hipótesis de que las instituciones no despliegan efectos por sí solas sino en conexión con otras.

La juventud se encuentra inmersa en el punto de gravedad del "triángulo de las interacciones" (Sabato y Botana, 1975, p. 146), entre las políticas públicas del Estado, la generación de conocimiento de la Academia -el cual, hasta hace poco, no trascendía al común de la sociedad latinoamericana (Duque, 2011), a pesar de la vinculación exigida por la Ley Orgánica de Educación Superior- y las exigencias de talento humano del sector empresarial. Ayala Mora (2018) sostiene que desde la alfabetización, en el Ecuador casi no existen políticas de Estado, de modo que se debe delinear y ejecutar una política de Estado sobre educación superior, que implique al sistema educativo regular y a las universidades y, desde luego, a quienes emplean los productos que salen de la universidad. Por cuanto asimétricos y discrepantes, la "no-sincronización" entre los objetivos de la administración pública, de las instituciones académicas y de los sectores productivos limitan las posibilidades de activar el crecimiento económico, toda vez que "el problema se encuentra en una rigidez [de] las políticas educativas, al no poder abarcar de manera flexible las oportunidades y las necesidades laborales de los jóvenes" (Petter y Moreno, 2019, p. 278). La Organización Internacional del Trabajo va más allá, afirmando que "los jóvenes tienen problemas para encontrar empleo debido al desajuste entre las competencias obtenidas y las exigidas en el mercado laboral" (2012: 56). En sentido análogo Bonilla sugiere que "existe una desconexión entre lo que piensan quienes conciben los sistemas de enseñanza universitaria y lo que desean o proyectan quienes tienen los instrumentos de trabajo", y por ello se debe "insistir en la necesidad de que los conocimientos que se imparten estén vinculados a los requerimientos nacionales. [...] Eso significa que se debe producir conocimiento aplicable, que se transforme en necesidades de innovación que puedan vincularse a la productividad" (entrevista personal). Efectivamente, el ámbito de la educación superior debe dirigir sus procesos, a más de lograr la excelencia académica, a alcanzar "un mayor impacto tanto económico como social y atemperarse a las demandas de la sociedad" (Abad, López y Fernández , 2017: 52), y “dar respuesta a las exigencias del mundo del trabajo, lo que significa que las universidades deben estar informadas de las expectativas y demandas de la sociedad, [...] preparando los profesionales que esta necesita en cada momento y en 
cada sector" (García-Blanco y CárdenasSempértegui, 2018: 328).

\section{Marco normativo}

En concordancia con los preceptos constitucionales 39 y 284.6 y 7 , el artículo 15 de la LJ establece como objetivos de las políticas públicas de promoción del empleo juvenil la creación de oportunidades de trabajo dirigidas a la población joven, $y$ el fomento del desarrollo de pasantías remuneradas, vinculadas a la formación profesional, entre otros. Como es sabido, con el objeto de aterrizar estas disposiciones programáticas, se expidió la Ley Orgánica para la Promoción del Trabajo Juvenil-LOPTJ (Asamblea NacionalRepública de Ecuador,2016). En su artículo tercero se define la noción de trabajo juvenil, que delimita como sujetos pasivos del contrato de trabajo juvenil a los jóvenes de entre 18 y 26 años. Ahora bien, resulta cuando menos llamativo que el legislador redujera en tres años el rango de edad de los sujetos pasivos del trabajo juvenil (LOPTJ) con respecto a la definición de joven según la LJ. Surge cierta confusión en cuanto a las distintas definiciones de joven y su discontinuidad en la legislación ecuatoriana y las contingencias públicas. Mientras Naciones Unidas considera jóvenes a las personas con edades comprendidas entre los 15 y los 24 años (Res. 36/28, 1981), y la LJ a todas las personas con edades entre 18 y 29 años (Art. 1), la LOPTJ excluye del contrato de trabajo juvenil a los jóvenes de entre 26 y 29 años.

Ciertamente la cobertura jurídico- normativa de la juventud del Ecuador es mayor que el estándar internacional. Sin embargo, la exclusión de los jóvenes de 26 a 29 años del trabajo juvenil -el cual persigue "impulsar el empleo juvenil [...], en condiciones justas y dignas"constituye un incumplimiento en materia laboral del principio pro homine, establecido en el artículo 3 de la LJ, y un tratamiento desigual en la implementación de políticas públicas conducentes a la garantía estatal del artículo 39 de la Constitución. El Plan Nacional de Desarrollo vigente pretendía reducir al $7,6 \%$ la tasa de desempleo juvenil para el año $2021^{15}$ (SENPLADES, $2017)^{16}$. Para ello recurre a la definición dada por la LJ, desplegando apriorísticamente la mayor protección estatal posible en atención a la normativa en materia juvenil, pero persiste la inadecuación del rango de edades de la LOPTJ, esto es, la exclusión de las personas de entre 26 y 29 años de las políticas de trabajo joven.

\section{Objetivos de desarrollo sostenible}

La cuestión que aquí se está tratando guarda estrecha relación con el $8^{\circ}$ ODS de Naciones Unidas, mediante el cual se

\footnotetext{
15 Incluso con anterioridad a la crisis sanitaria la consecución de la meta del ejecutivo parecía inalcanzable, ya que a pesar de la bajada de la tasa hasta el 8,3\% en 2018 (Secretaría Técnica Planifica Ecuador, 2019), en diciembre de 2019 esta repuntó hasta el 9\% (INEC, 2019b).

${ }^{16}$ Según el tabulado de la ENEMDU de 2019, en diciembre de dicho año la tasa de desempleo en personas de entre 15 y 24 años, y 25 y 34 , fue de $37,6 \%$ y $29,6 \%$ respectivamente (INEC, 2019b). A más de la inconsistencia conceptual entre la LJ y la LOPTJ, y la discontinuidad entre las variables clasificatorias de las ENEMDU 2018 y 2019 -como se apunta en la introducción-, se observa que el INEC no permite mayor conocimiento estadístico de la cuestión juvenil por cuanto no delimita los rangos de edad según la LJ.
} 
pretende "promover el crecimiento económico sostenido, inclusivo y sostenible, el empleo pleno y productivo y el trabajo decente para todos", para cuyo cumplimiento se antoja imprescindible la generación de "oportunidades laborales para toda la población en edad de trabajar, con condiciones de trabajo decentes [...]; asimismo, el aumento de la productividad laboral, la reducción de la tasa de desempleo, especialmente entre los jóvenes [...]" (ONU, 2018, p. 39).

Para verificar el compromiso de los Estados, Naciones Unidas instauró una serie de herramientas de seguimiento y evaluación. Por cercanía temporal, resulta especialmente importante la meta sexta: "de aquí a 2020, reducir considerablemente la proporción de jóvenes que no están empleados y no cursan estudios ni reciben capacitación" (2018, p. 40).

Como puede observarse, el plazo de vencimiento de esta meta expiró a comienzos del año 2020. Bien es verdad que, por cuanto genérica, su redacción es ambigua -reducir considerablemente-, toda vez que no precisa hasta qué punto debe reducirse la proporción de jóvenes desempleados y/o que no estudian. Según calcularon Buitrón, Jami y Salazar (2018, p. 54) en base a las estadísticas del INEC, en 2014 el 18,5\% de jóvenes ecuatorianos ni estudiaban ni trabajaban, porcentaje inferior al 23,6\% de jóvenes que "no estudian, no trabajan" bajo criterio de la extinta Secretaría Técnica de Juventudes (2018, p. 11). Mientras sí es posible determinar una cifra aproximada de los jóvenes desempleados, se desconoce el número de jóvenes matriculados en el conjunto de IES, ya que el último boletín analítico de la SENESCYT, publicado en diciembre de 2018, no aporta datos de matriculación en universidades y escuelas politécnicas más allá de 2016 (SENESCYT, 2018a), si bien el informe de cifras nacionales sugiere una tasa de matriculación bruta y neta del 30\% y del $22,2 \%$ respectivamente (SENESCYT, 2018b). Ello impide auscultar con criterios cuantitativos el nivel de cumplimiento del Estado ecuatoriano en la meta sexta del ODS $8^{\circ}$, aunque, con todo, las últimas cifras oficiales -a la espera aún del primer reporte de 2020 de la ENEMDU- indican que se logró un descenso de $1,4 \%$ en la tasa de desempleo juvenil de 18 a 29 años (INEC, 2019b), si bien la proyección de desempleo juvenil para 2020, realizada por la SENESCYT en base a la data del INEC, era del 9,5\% (SENESCYT, 2018b).

El $8^{\circ}$ ODS posee fuertes nexos con sus homólogos $4^{\circ}, 5^{\circ}$, y $10^{\circ}$ (ONU, 2018). Es frecuente leer en la literatura especializada y en los lineamientos internacionales las bondades de la educación frente a la exclusión social, la inequidad y la desigualdad de género (Pérez, 2008; Comité Económico y Social Europeo, 2010; Muñoz, 2010; UNESCO, 2012). Así, el ODS $8^{\circ}$ plantea una lectura polivalente y holística, interconectada con los objetivos $4^{\circ}, 5^{\circ}$ y $10^{\circ}$.

\section{Metodología}

Esta investigación no persigue el levantamiento de información estadística 
nueva, la realización de mediciones o la validación de resultados numéricos, sino el análisis del conjunto de estadísticas base.

De un lado, esta investigación se cimienta sobre el análisis cuantitativo de los reportes de la ENEMDU publicados por el INEC (2018, 2019a y 2019b), las estadísticas laborales (INEC, 2017a y 2017b) y las estadísticas de la SENESCYT (2018a, 2018b). La ENEMDU permite conocer los niveles de empleo adecuado, por rango de edades, nivel de instrucción académica, género y etnia, mientras que del boletín de la SENESCYT se desprende la desigualdad étnica en el acceso a la educación superior. Sin embargo, por cuanto la metodología se asienta en estadísticas oficiales -las únicas a nivel nacional que han estudiado con criterios científicos la relación entre empleo y nivel de instrucción-, esta es intrínsecamente limitada, habida cuenta que las categorías estudiadas por la ENEMDU han variado de un año a otro y los rangos de edad manejados por el INEC no se corresponden con la delimitación normativa del término "joven" (como ya se detalló), impidiendo la ubicación del espectro etario juvenil -sea siguiendo el parámetro de la LJ o el de la LOPTJ- en una categoría estadística específica.

Con la finalidad de aportar distintas circunspecciones cualitativas - interpretativas frente a la cuestión del empleo juvenil y su relación con la educación superior $\mathrm{y}$, a su vez, con las políticas curriculares de las IES, se añaden dos opiniones receptadas en sendas entrevistas. La primera entrevista fue realizada a Adrián Bonilla el 16 de octubre de 2018, en calidad de máxima autoridad de la SENESCYT, cargo que ocupó desde agosto de 2018 hasta mediados de 2019. Dicha entrevista es relevante por cuanto deja conocer las impresiones y las preocupaciones sobre acceso a la educación y absorción laboral del titular de la institución pública encargada de la educación superior. La segunda entrevista se realizó el 19 de octubre de 2018 al académico ecuatoriano Enrique Ayala Mora, Doctor en Educación y profundo conocedor de la situación histórica y actual de la educación superior nacional. También relevante, en esta entrevista se habló sobre la capacidad de las IES de combatir las cifras juveniles de desempleo y empleo no adecuado.

\section{Resultados}

Los jóvenes representan el 20\% de la población ecuatoriana (Secretaría Técnica de Juventudes, 2018), pero solo el $35 \%$ de los jóvenes con títulos de educación superior posee un empleo adecuado (INEC, 2019b). En mayo de 2019 el número total de títulos de educación superior registrados en la SENESCYT era 2.174.206 -a lo que se debe sumar los 391.642 títulos técnicos y tecnológicos inscritos tras la reforma del artículo 118 de la LOES (SENESCYT, 2019)-. Ahora bien, se sabe que con fecha de corte 5 de diciembre de 2018 había 2.112.835, de los cuales el 68\% correspondían a tercer nivel (SENESCYT, 2018a). Esto es, entre diciembre de 2018 y mayo de 2019, hubo un incremento de 61.371 registros de títulos superiores, 
precisamente en un contexto de deterioro de la calidad del trabajo y retracción económica.

Partiendo de la consideración general de los jóvenes como futuro de la sociedad, las tasas de desempleo y subempleo juvenil de los últimos años resultan alarmantes. De ahí que, a partir de la segmentación laboral y el auge de una economía más especializada con componentes socioeconómicos más específicos, los paradigmas tradicionales que han venido defendiendo la relación orgánica entre educación superior y empleo amenazan con derrumbarse. El desempleo joven no es una problemática aislada. Al contrario, conforma ramificaciones multidimensionales extensibles al conjunto de experiencias sociales de los afectados. Más cuando se descubre que muchos de los jóvenes desempleados, y/o sus familias, han invertido sumas considerables o se han endeudado para costear los estudios. Poco a poco, la educación de tercer nivel comienza a revelarse como insuficiente para acceder al mercado laboral más competitivo que se recuerde (como se observa en el conjunto de estadísticas del INEC y la SENESCYT planteadas, la especialización y las capacidades técnicas han aumentado progresivamente durante el último lustro). Con el incremento del registro de títulos de cuarto nivel, que pasaron del $2,9 \%$ al $4,7 \%$ durante la década pasada (INEC, 2017b), las expectativas laborales de quienes no cuentan con posgrados se complican, convirtiendo tácitamente la posesión de títulos de cuarto nivel como nuevo requisito de contratación.
En términos sociológicos, el desempleo y el subempleo juvenil sobredimensionan los males endémicos de la región -pobreza, inequidad, precariedad laboral, dicotomía urbano-rural, etc.-, por cuanto coartan las posibilidades de la educación como motor de inclusión socioeconómica y de superación de las desigualdades, así como contribuyen a la "transmisión intergeneracional de la pobreza" (Solon, 1992, p. 397). Según Jacinto,

Durante décadas, la integración social de los jóvenes se canalizó a través de las instituciones educativas $\mathrm{y}$ de las ligadas al mundo productivo [...]. Este modelo de inserción entre la educación y el trabajo o entre el mundo familiar y el trabajo -según el sector social de origen- se va rompiendo en el marco de la crisis del empleo, para convertirse en una transición larga y compleja. (2006, p. 68-69).

La actual situación laboral impide a los jóvenes aspirar a una estabilidad mínima que les permita edificar sus proyectos de emancipación y maduración. La cuestión profesional, en el contexto de la educación superior, es socialmente relevante como elemento constitutivo de la identidad de los sujetos. El empleo es una esfera nuclear, no solo en términos de crecimiento económico, sino de desarrollo humano y sus expectativas de futuro, las cuales se ven mermadas ante el complejo acceso al mercado laboral.

En los últimos años el Estado ha tomado varias medidas en aras de facilitar la inserción laboral juvenil. Pese 
a su irregular acogida, el ejemplo paradigmático es la iniciativa "Mi Primer Empleo" (2007), la cual en 2017 únicamente contabilizó 774 beneficiados de un total de 15.365 jóvenes inscritos (Ministerio del Trabajo, 2017a). Para 2018 se reestructuró el proyecto con un presupuesto actualizado de más de treinta millones de dólares y un plazo de ejecución hasta 2021 (Ministerio del Trabajo, 2017b), extendiendo el rango de las prácticas pre-profesionales a una red de aliados privados según convenios de cooperación pública-privada y con las IES -en la actualidad se cuentan más de 4.180 beneficiados y 849 convenios (Ministerio del Trabajo, 2020)-. Sin embargo, ya en septiembre de 2019 se indicó que el número de beneficiados publicitado por el Estado se circunscribía al 20\% de jóvenes ubicados en prácticas o pasantías (Torres, 2019). Ciertamente, la realización de prácticas y pasantías remuneradas puede llegar a ser muy positivo para los jóvenes, no obstante, llama la atención que la evaluación de resultados e impacto del proyecto solo muestra interés por los convenios y la realización de pasantías, de las cuales más de un tercio se proyectan en el sector público (Ministerio del Trabajo, 2017b). Esto es, la generación de empleo joven no figura entre sus elementos de evaluación. Por otro lado, en reconocimiento de la complejidad de la realidad juvenil, el actual gobierno creó la ya extinta Secretaría Técnica de Juventudes, cuyo único proyecto destacable fue "Impulso Joven" -con apoyo de BanEcuador-, financiando 267 emprendimientos juveniles, capacitando a 6.087 jóvenes y colocando en 2018 créditos por valor de 289,8 millones de dólares (Secretaría Técnica Planifica Ecuador, 2019) ${ }^{17}$.

En esta tentativa de triángulo de Sabato se desliza el esfuerzo público por generar una interconexión entre los sectores productivos, la Academia y el Estado. Ahora bien, el Ministerio del Trabajo no ha publicado información actualizada sobre el acceso real al empleo -no pasantías, ni prácticas- derivado de "Mi Primer Empleo". No se puede hablar, por lo tanto, de una política pública eficiente, ya que no posee una criteriología de evaluación clara. Tampoco permite cotejar los datos con las estadísticas levantadas por otras instituciones del Estado, ya que se rigen por variables dispares.

En opinión de Ayala Mora (2018), la política de Estado que reclama en materia de educación superior no debe ignorar la autonomía de las IES, lo único que el Estado puede hacer, más bien, es "incentivar la relación universidadempresa [...], y crear canales de relación entre el sector productivo y la universidad, lo cual solo se puede hacer profundizando la autonomía de esta". Defiende, en definitiva, un cambio de modelo ajeno a la "dictadura de la planificación", ya que "una cosa es que las políticas que rigen el país sean obligatorias, pero otra es que el Plan de Desarrollo que aprueba cada gobierno se

\footnotetext{
${ }^{17}$ Cotejando la suma monetaria y el número de emprendimientos reflejados en el informe, el costo de cada emprendimiento refleja una inversión media de más de un millón de dólares. No se ha podido encontrar un documento oficial que refleje los planes de viabilidad de los emprendimientos, los criterios para el otorgamiento de los créditos, la media de las cantidades entregadas ni los plazos de retorno.
} 
vuelva obligatorio para la universidad". Lo que se propone, en resumidas cuentas, es el respeto efectivo de la autonomía universitaria para generar programas académicos conjuntamente el sector privado, de beneficio mutuo y que posibilite a las IES diseñar programas propios reconocidos por la SENESCYT en los que la empresa posea mayor protagonismo en la formación de sus futuros profesionales.

En este sentido, "las experiencias mundiales son diversas, y en todas ellas se parte de una autonomía irrefutable y de una disponibilidad de los sectores empresariales para aportar" (Ayala Mora, 2018). Y como expresó Bonilla (2018), es responsabilidad de la Academia "producir conocimiento aplicable". La cuestión del desempleo juvenil está atravesada por una profunda holística. Es una arista más del desempleo general y la falta de inversión privada en tiempos de contracción económica. Entonces, ¿cómo generar atractivo productivo?, ¿y cómo podrían darse las relaciones universidadempresa que reclaman Bonilla (2018) y Ayala Mora (2018)? Van Breugel (2016) afirma que lo que más valoran los empleadores es la experiencia y la capacitación profesional simultánea a la preparación académica. Ya en 2011 la OIT recomendó "vincular la experiencia laboral y la participación de los jóvenes al sector privado, a través de la formación en la empresa, [ya que] aumentan las oportunidades de empleo" (2011, p. 4). Ante la problemática, la mayoría de las IES carecen de estudios de base que posibiliten un enfoque profesionalizante -abierto lo teórico hacia la propia praxis- en los diseños curriculares, desatendiendo las necesidades genéricas o coyunturales que enfrentan los entes generadores de empleo.

Por citar algunos ejemplos, esta idea la siguieron los modelos estadounidense, chileno y español, persiguiendo la generación de relaciones sinergéticas entre la Academia y el sector privado, y brindando un cariz profesionalizante a la educación acorde a las conocidas competencias de empleabilidad. Sin ir más lejos, en Chile se ha venido implementando desde 1995 un sistema educativo dual, voluntario y gradual de formación técnica y profesional, compartida entre las instituciones educativas y la empresa (OEI, 2011). Posiblemente el caso de estudio por antonomasia sobre vinculación AcademiaEmpresa sea el clúster formado por la Universidad de California y la industria vinícola californiana (Mueller y Sumner, 2006; Hira y Swartz, 2014). En él la administración pública estatal y el sector privado financian el Department of Viticulture \& Enology, dedicado a la investigación y a la implementación de sus hallazgos en los viñedos de las empresas financistas, mientras que la Universidad ofrece programas de grado y posgrado según sus propios lineamientos académicos e investigativos y los requerimientos en materia de talento humano y competencias laborales del sector privado. Otros buenos ejemplos de relaciones académicas-empresariales se pueden encontrar en la Universidad de Navarra, la Universidad Nebrija y la Universitat Internacional de Catalunya, poseedoras todas de iniciativas 
fundamentadas en un modelo de alianzas entre las casas de estudios y el sector privado en base a intereses comunes de investigación, formación del alumnado y crecimiento sinergético.

Los estudios de saturación preferencial determinaron que la demanda estudiantil ecuatoriana se inclinó por estudiar, en este orden, medicina, enfermería, odontología, administración y derecho (Bonilla, 2018). Pero ¿hasta qué punto están alineadas estas preferencias con la economía nacional? En 2018 el 29,4\% del empleo se concentró en la agricultura, ganadería y pesca, carreras con tasas de postulación muy bajas, mientras que el $64 \%$ de postulantes se concentraron en programas cuyas opciones de empleo no sobrepasan conjuntamente el $4,3 \%$ del mercado laboral (SENESCYT, 2018c). Se comprueba que las carreras de ciencias sociales son escogidas en un $45 \%$, ingenierías un $14 \%$ y las carreras del área de salud un 13,6\% (SENESCYT, 2018a). Según el Ministerio de Educación (2020) se ha mantenido esta tendencia. Ello demuestra un desbordamiento y una concentración considerable en la demanda de educación superior ${ }^{18}$, dándose asimetría entre el número anual

\footnotetext{
${ }^{18}$ A diciembre de 2018 se registraban 2.112 .835 títulos, de los cuales $87 \%$ son nacionales (SENESCYT, 2018a). El registro de títulos extranjeros aumentó del 4\% al 13\% entre 2015 y 2018. Nótese la problemática, tanto a nivel particular como público, de la falta de empleo adecuado en quienes hayan cursado sus estudios en el extranjero. Lamentablemente, no hay datos que relacionen empleo y educación internacional. Bonilla (2018) reconoce la existencia de "algunas generaciones de becarios que el Estado financió sus estudios en el exterior, que no necesariamente llegan y les está esperando un cargo o un empleo en su especialidad", a la vez que recomienda a las nuevas generaciones pensar en opciones de estudio ajenas a las carreras con mayor saturación preferencial.
}

de graduados y el incremento de puestos de trabajo, produciéndose el conocido cuello de botella en el acceso laboral.

Tampoco parece que los jóvenes bachilleres dispongan de información oficial consolidada sobre tasas de inserción laboral, empleabilidad, competencia dentro de las distintas carreras o salidas profesionales. La concepción de la problemática se acrecienta al comprobar que, según la última data disponible, la tasa de emprendimiento joven es del $18 \%$ (Secretaría Técnica de Juventudes, 2018), presentando gran dependencia a la generación de empleo externo.

\section{Conclusiones}

Ciertamente, el desempleo y el subempleo juvenil responden a cuestiones sistémicas y ciclos económicos. Como se ha probado, incluso antes de la crisis sanitaria, el panorama nacional en materia de empleo juvenil no invitaba al optimismo. En diciembre de 2019 -apenas un mes antes de alcanzar la fecha establecida para mostrar avances en el ODS 8.6-, la tasa de desempleo juvenil todavía se situaba un $1,4 \%$ por encima de la meta proyectada (SENPLADES, 2017; INEC, 2019b). En este contexto, los jóvenes con titulación superior no están necesariamente más protegidos del desempleo y el subempleo que aquellos que carecen de títulos superiores. Así lo evidencia el contraste entre la tendencia alcista de matriculación bruta en IES y registro de títulos en la SENESCYT y el escaso $35 \%$ de jóvenes que, durante 2019, gozaron de un empleo adecuado. 
Se comprueba, además, la saturación preferencial de determinadas carreras, provocando un cuello de botella entre el número de jóvenes titulados que cada año ingresan al mercado laboral y la asimétrica creación de puestos de trabajo.

Para futuras investigaciones, se sugiere relacionar mallas curriculares, competencias específicas, perfiles de egreso y tasas de empleabilidad con la existencia de experiencias compartidas entre las IES y los sectores productivos. Arrojaría luz al respecto ahondar en los requerimientos de talento humano de los diversos segmentos laborales, especificando las competencias más valoradas por los empleadores $\mathrm{y}$, concretamente, el rol actual o potencial que, en el desarrollo y perfeccionamiento de estas, poseen las IES. No obstante, aún consolidando data del sector privado, las incongruencias entre las distintas herramientas oficiales de medición, tal y como están diseñadas en la actualidad, dificultarían la lectura integral de las dinámicas laborales en la franja etaria joven y su relación con el componente educativo.

\section{Agradecimiento}

A Gisela Montalvo, Directora de la Escuela de Gobierno del IDE Business School, y a Daniel Barragán, Director del Centro Internacional de Investigaciones sobre Ambiente y Territorio - CIIAT.

\section{Referencias}

Abad, C., López, M., y Fernández, K. (2017). El sistema de educación superior ecuatoriano visto desde los principios de pertinencia y calidad. Universidad y Sociedad, 9(5), 46-53.

Asamblea Nacional-República de Ecuador. (2016) Ley Orgánica para la Promoción del Trabajo Juvenil (LOPTJ).

ASOBANCA. (2020a). Boletín macroeconómico. Enero 2020.

ASOBANCA. (2020b). Boletín macroeconómico. Marzo 2020

Ayala Mora, E. (19 de octubre de 2018). Entrevista personal.

BCE. (2020). Previsiones macroeconómicas 2020 (1).

BID. (2017). Compás Millennial. La generación Y en la era de la integración 4.0. (Nota Técnica No. IDB-TN-1283).

Brzinsky-Fay, C. (2017). The Interplay of Educational and Labour Market Institutions and Links to Relative Youth Unemployment. Journal of European Social Policy, 27(4), 346-359. doi: https://doi.org/10.1177/095 8928717719198.

Bonilla, A. (16 de octubre de 2018). Entrevista personal.

Buitrón, K., Jami, V., y Salazar, Y. (2018). Los jóvenes ninis en el Ecuador. Revista de Economía del Rosario, 21 (1), 39-80. doi: http://dx.doi.org/10.12804/revistas.urosar io.edu.co/economia/a.6800.

Cano, A. (2014). Juventud, trabajo y desempleo en los prolegómenos de la crisis económica en España. Reflexiones críticas. Acta Sociológica, 63, 99-120. doi: https://doi.org/10.1016/ S0186-6028 (14)70484-0.

Comité Económico y Social Europeo. (2010). Informe de la Conferencia Bienal 2010. La educación para luchar contra la exclusión social. Recuperado de: 
https://www.eesc.europa.eu/resources/do cs/biennal-full-report-es.pdf.

De Ibarrola, M. (2005). Educación y trabajo. Revista Mexicana de Investigación Educativa, 10 (25), 303-313.

Deloitte. (2019). Global Millennial Survey 2019. Recuperado de: https://www2.deloitte.co $\mathrm{m} /$ global/en/pages/about-deloitte/articles/ millennialsurvey.html.

Duque, J. (2011). Los usos sociales del conocimiento. A propósito de las relaciones universidad-sociedad. Administración \& Desarrollo, 39(53), 7-22.

Espinosa, B., y Esteves, A. (2006). Expectativas y estrategias laborales de los jóvenes en Quito. En L. Martínez Valle (ed.), Jóvenes y mercado de trabajo en el Ecuador (pp. 103-126). FLACSO, CEPAL, Deutsche Gesellschaft für Technische Zusammenarbeit.

Fernández Enguita, M. (1992). Educación, formación y empleo. España: Eudema Universidad.

Ferreyra, M., Avitabile, C., Botero Álvarez, J., Haimovich, F., y Urzúa, S. (2017). At a Crossroads. Higher Education in Latin America and the Caribbean. Washington D.C.: World Bank. doi: https://doi.org/ 10.1596/978-1-4648-1014-5.

García-Blanco, M., y Cárdenas-Sempértegui, E. (2018). La inserción laboral en la Educación Superior. La perspectiva latinoamericana. Educación XX1, 21(2), 323-347. doi: 10.5944/educXX1.16209.

González Arratia, N. I., y Valdez Medina, J. L. (2012). Optimismo-pesimismo y resiliencia en adolescentes de una universidad pública. Ciencia Ergo Sum, 19(2), 207-214.

Guerrero, A. (1999). El enfoque de las competencias profesionales: una solución conflictiva a la relación entre formación y empleo. Revista Complutense de Educación,
10(1), 335-360.

Hira, A., y Swartz, T. (2014). What makes Napa Napa? The Roots of Success in the Wine Industry. Wine Economics and Policy, 3, 37-53. doi: http://dx.doi.org/10.1016/j.we p.2014.02.001.

INEC. (2017a). Panorama Laboral y Empresarial del Ecuador, 2017.

INEC. (2017b). Determinantes de la movilidad laboral en Ecuador 2006-2017. Un análisis del empleo registrado en la seguridad social. Recuperado de https://www.ecuadorencifras.gob.ec/docu mentos/web-inec/boletin/Presentaciones Seminario_Sec_Lab/Determinantes_de_1 a_movilidad_laboral_en_Ecuador.pdf.

INEC. (2018). Encuesta Nacional de Empleo, Desempleo y Subempleo Acumulada. Indicadores de mercado laboral 2018. Recuperado de https://www.ecuadoren cifras.gob.ec/documentos/web-inec/Sitio s/ENEMDU_ACUMULADA/index.html.

INEC. (2019a). Encuesta Nacional de Empleo, Desempleo y Subempleo. Indicadores laborales diciembre 2019. Recuperado de https://www.ecuadorencifras.gob.ec/docu mentos/web-inec/EMPLEO/2019/Diciem bre/201912_Mercado_Laboral.pdf.

INEC. (2019b). Encuesta Nacional de Empleo, Desempleo y Subempleo. Información estadística. Tabulados y series históricas. Recuperado de https://www.ecuadorenci fras.gob. ec/empleo-diciembre-2019/.

INEC. (2019c). Directorio de Empresas y Establecimientos 2018.

Jacinto, C. (2006). Los caminos de América Latina en la formación vocacional de jóvenes en situación de pobreza. Balance y nuevas estrategias. En M. De Ibarrola (coord.), Desarrollo local y formación: hacia una mirada integral de la formación de los jóvenes para el trabajo (pp. 67-102). 
Centro Interamericano para el Desarrollo del Conocimiento en la Formación Profesional, Organización Internacional del Trabajo.

Jiménez, A. (2009). Reflexiones sobre la necesidad de acercamiento entre universidad y mercado laboral. Revista Iberoamericana de Educación, 5(1).

Londoño, C. (2009). Optimismo y salud positiva como predictores de la adaptación a la vida universitaria. Acta Colombiana de Psicología, 12(1), 95-107.

Márquez, A. (2011). La relación entre educación superior y mercado de trabajo en México. Perfiles Educativos, 33, 169-185.

Ministerio de Educación. (2020). Lista de carreras universitarias con mayor demanda. Recuperado de https://educacionecuador ministerio.blogspot.com/2018/02/lista-de -carreras-universitarias-ecuador-mas-de mandadas.html.

Ministerio del Trabajo. (2017a). Informe Técnico de Gestión. Proyecto Mi Primer Empleo, Año 2017. Recuperado de http://www.tra bajo.gob.ec/wp-content/uploads/2018/03/ INFORME-2017-MI-PRIMER-EMPLE O.pdf.

Ministerio del Trabajo. (2017b). Proyecto Mi Primer Empleo. Periodo 2007-2021. Recuperado de http://www.trabajo.gob.ec /wp-content/uploads/2019/01/Document o-Proyecto-Mi-Primer-Empleo.pdf.

M\&C Saatchi. 2018. Risk, Realism and Ritalin. A study of the Post-Millennial Generation. Recuperado de http://mcsaatchitransform. com /src/assets/pdfs/MCTRANSFORM RRR.pdf.

Mueller, R. A., y Sumner, D. (2006). Clusters of Grapes and Wine. $3^{\circ}$ International Wine Business Research Conference. Recuperado de https://www.agmrc.org/media/cms/Wi ne_Clusters2_84CD1EE476398.pdf.
Mungaray, A. (2001). La educación superior y el mercado de trabajo profesional. Revista Electrónica de Investigación Educativa, 3(1), 54-66.

Muñoz, V. (2010). El derecho a la educación: algunos casos de exclusión y discriminación. Revista Instituto Interamericano de Derechos Humanos, 52, 267-308.

Mullo, H., y Marcatoma, J. (2018). Desempleo juvenil Ecuador, 2017: magnitud y construcción de perfiles sociodemográficos. Revista Caribeña de Ciencias Sociales, (Marzo), 1-9.

OECD. (2015). Education at a Glance Interim Report: Update of Employment and Educational Attainment Indicators. Recuperado de https://www.oecd.org/edu cation/eag-interim-report.pdf.

OECD. (2017). Educational attainment: A snapshot of 50 years of trends in expanding education. Recuperado de https: //www.oecd-ilibrary.org/docserver/409ce b2b-en.pdf?expires $=1590538685 \& \mathrm{id}=\mathrm{id}$ $\&$ accname $=$ guest $\&$ checksum $=84$ C9DC 1 684CD908EB36E1DADD3AD4CCA.

OEI. (2011). Inserción laboral de los jóvenes. Estrategias innovadoras para facilitar la transición escuela-trabajo.

OIT. (2011). Políticas de empleo juvenil durante la recuperación económica. Recuperado de https://www.ilo.org/global/docs/WC MS_151462/lang--es/index.htm.

OIT. (2012). La crisis del empleo de los jóvenes: iActuemos ya! Conferencia Internacional del Trabajo, Reunión No. 101.

Olmedo, P. (2018). El empleo en el Ecuador. Una mirada a la situación y perspectivas para el mercado laboral actual. FriedrichEbert-Stiftung, ILDIS.

ONU. (2018). La Agenda 2030 y los Objetivos de Desarrollo Sostenible: una oportunidad para América Latina y el Caribe 


\section{(LC/G.2681-P/Rev.3).}

Pérez, C. (2008). La educación contra la exclusión. Eikasia, Revista de Filosofia, 3(17), 243-260.

Petter, L. y Moreno, C. (2019). Subempleo en el mercado laboral juvenil en Ecuador. Revista nuestrAmérica, 7(13), 265-280.

Planas Coll, J., y Enciso Ávila, I. (2014). Los estudiantes que trabajan: ¿tiene valor profesional el trabajo durante los estudios? Revista Iberoamericana de Educación Superior, 5(12), 23-45. doi: https://doi.org/10.1016/S2007-2872 (14)71941-9.

Quispe, G., Sánchez, L., y Arellano, O. (2019). Los costos del contrato juvenil en las empresas industriales del Ecuador. Cumbres, 5(1), 55-68.

Sabato, J. A., y Botana, N. (1975). La ciencia y la tecnología en el desarrollo futuro de América Latina. En J. A. Sabato (comp), El pensamiento latinoamericano en la problemática-ciencia-tecnología-desarro llo-dependencia (pp. 143-154). Buenos Aires: Editorial Paidós.

SENESCYT. (2018a). Boletín analítico de educación superior, ciencia, tecnología, innovación $y$ saberes ancestrales. Recuperado de https://www.educacionsuperior.gob.ec/w p-content/uploads/downloads/2019/01/B oletin_Analitico_SENESCYT_Diciembr e-2018.pdf.

SENESCYT. (2018b). Educación superior, ciencia, tecnología, innovación y saberes ancestrales en cifras. Recuperado de https://www.educacionsuperior.gob.ec/cif ras-a-nivel-nacional-y-provincial-de-la-o ferta-academica-acceso-y-permanencia-e n-el-sistema-de-educacion-superior/.

SENESCYT. (2018c). Carreras necesarias para el desarrollo del país y con alto nivel de empleabilidad tienen poca demanda (Boletín No. 095). Recuperado de https://www.educacion superior.gob.ec/ carreras-necesarias-para-el-desarrollo-del -pais-y-con-alto-nivel-de-empleabilidad-t ienen-poca-demanda/.

SENESCYT. (2019). 391.642 títulos técnicos y tecnológicos ya están registrados como tercer nivel (Boletín No. 185). Recuperado de https://www.educacionsu perior.gob.ec/391-642-titulos-tecnicos-ytecnologicos-ya-estan-registrados-como-t ercer-nivel/.

SENPLADES. (2017). Plan Nacional de Desarrollo 2017-2021. Toda una Vida.

Secretaría Técnica de Juventudes. (2018). DieciochoVeintinueve en números. Recuperado de http://www.juventudes.gob.ec/dieciochoveintinueve/.

Secretaría Técnica Planifica Ecuador. (2019). Informe de avance del cumplimiento de la Agenda 2030 para el desarrollo sostenible.

Seligman, M. (1999). Niños optimistas. Cómo prevenir la depresión en la infancia. Barcelona: Grijalbo.

Solon, G. (1992). Intergenerational Income Mobility in the United States. The American Economic Review, 82(3), 393-408.

Torres, W. (3 de septiembre de 2019). Mi Primer Empleo ubica a dos de cada 10 postulantes en prácticas o pasantías. Primicias. Recuperado de https://www. primicias.ec/noticias/economia/primer-e mpleo-solo-ubica-uno-cada-diez/.

UNESCO. (2012). Lucha contra la exclusión en la educación. Guía de evaluación de los sistemas educativos rumbo a sociedades más inclusivas y justas.

Van Breugel, G. (2016). Formación profesional y capacitación: revisión de experiencias en países desarrollados. En A. Isgut \& J. 
Weller (ed.). (2016). Protección y formación. Instituciones para mejorar la inserción laboral en América Latina y Asia (pp. 219-253). CEPAL.

Zambrano, J., y Lasio, V. (2019). Jóvenes emprendedores en Ecuador 2012-2017. GEM Ecuador Reporte Especial. Recuperado de http://www.espae.espol. edu.ec/wp-content/uploads/documentos/g em_jovenes_2012_2017.pdf. 